\title{
NONLINEAR ANALYSIS OF PLANE FRAMES USING A COROTATIONAL FOMULATION AND PLASTICITY BY LAYERS IN A TIMOSHENKO BEAM EL- EMENT
}

\author{
Sebastião Simão da Silva, William Taylor Matias Silva
}

Department of Civil and Environmental Engineering, Postgraduate Program in Structure and Civil Construction Engineering, University of Brasília (sebastiaosim@unb.br)

\begin{abstract}
The purpose of this work is to perform a nonlinear analysis of plane frame structure using a corotational formulation and a layered plastic modeling. The plane frame is discretized with a 2D Timoshenko beam element. Plasticity is introduced by rate-independent Von-Mises model with isotropic hardening. Numerical integration over the cross-section is performed for obtain the internal force vector and tangent stiffness matrix of these elements. At each integration point, the backward-Euler algorithm is used for integration in the constitutive equations. Some examples are used in order to check the performances in the elements and the path-following procedures.
\end{abstract}

Keywords: Nonlinear analysis of structures; Corotational formulation; Plasticity in layers; Finite element method.

\section{INTRODUCTION}

The linear analysis presents difficulties for analysis the real behavior of unusual structures, when the loading conditions is not common, or in structures close to collapse. In this context, the nonlinear material and geometric has wide applicability in structural engineering.

In the geometric nonlinear analysis using finite element method, three different types of kinematic descriptions have been widely used: total lagrangian description, updated lagrangian description and corotacional description [10]. The latter is originates from the polar decomposition theorem which states that the total deformation of a solid surface can be decomposed into rigid body motion and relative deformation [6] - Figure 1.

The concept of kinematic description corotacional was introduced in a context FEM in the 60s of last century with the work of Argyris [1], is the latest of the formulations used in geometrically nonlinear analysis and has a wide variety of subjects to be investigated [5]. 


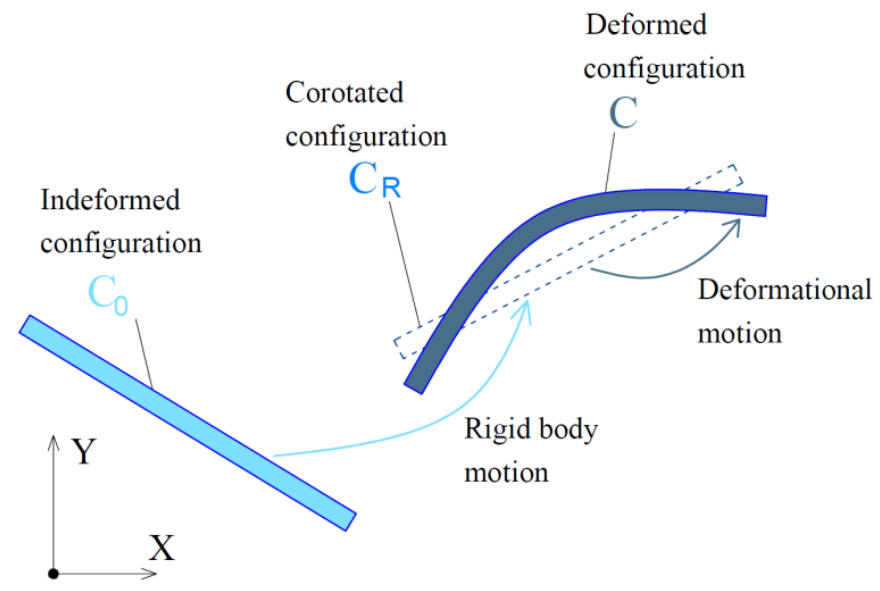

Figure 1. Kinematic description of the corotacional formulation.

When the answer in the solid structure is elastic, ceased loading, the body does not exhibit deformation. However, when the answer is plastic after ceased the loading the material shows residual strain [7][8]. The theory of plasticity provides laws and models capable of describing the constitutive behavior of materials with elastoplastic response.

\section{METHODOLOGY APPROACH}

In this work used a 2D Timoshenko beam element $\left(\mathrm{C}_{0}\right)$ without coupling of the axial and bending effort. To perform numerical simulations with the described formulation, implement in the finite element program 2D_Beam_f90 the beam elements mentioned above using the corotational formulation. In the treatment of plasticity has been used a layered unidimensional bilinear model with isotropic hardening. Integrates this model using an implicit algorithm named backward-Euler [11]. For the sectional efforts, integrate normal stresses using seven or fifteen Gauss points along the depth of the cross section. Vector of internal forces and tangent stiffness matrix of element mentioned above was determined through the principle of virtual work. The coefficients of these vectors and matrices were obtained by numerical integration. For the trajectory equilibrium nonlinear adopts an analysis based on the iterative incremental Newton-Raphson method and arc length technique.

\section{COROTATIONAL FORMULATION OF PLANES FRAMES}

The formulation described here is presented in Battini [2], which differs slightly from the other one shown in Crisfield [3].

\subsection{Kinematic description of a beam element}

In Fig 1 shows the coordinates of the nodes 1 and 2 in the global coordinate system $(x, z)-\left(x_{1}, z_{1}\right)$ and $\left(x_{2}, z_{2}\right)$, respectively (Figure 2$)$. The global displacement vector is defined by: 


$$
\boldsymbol{u}_{g}=\left\{\begin{array}{llllll}
u_{1} & w_{1} & \theta_{1} & u_{2} & w_{2} & \theta_{2}
\end{array}\right\}^{T} .
$$

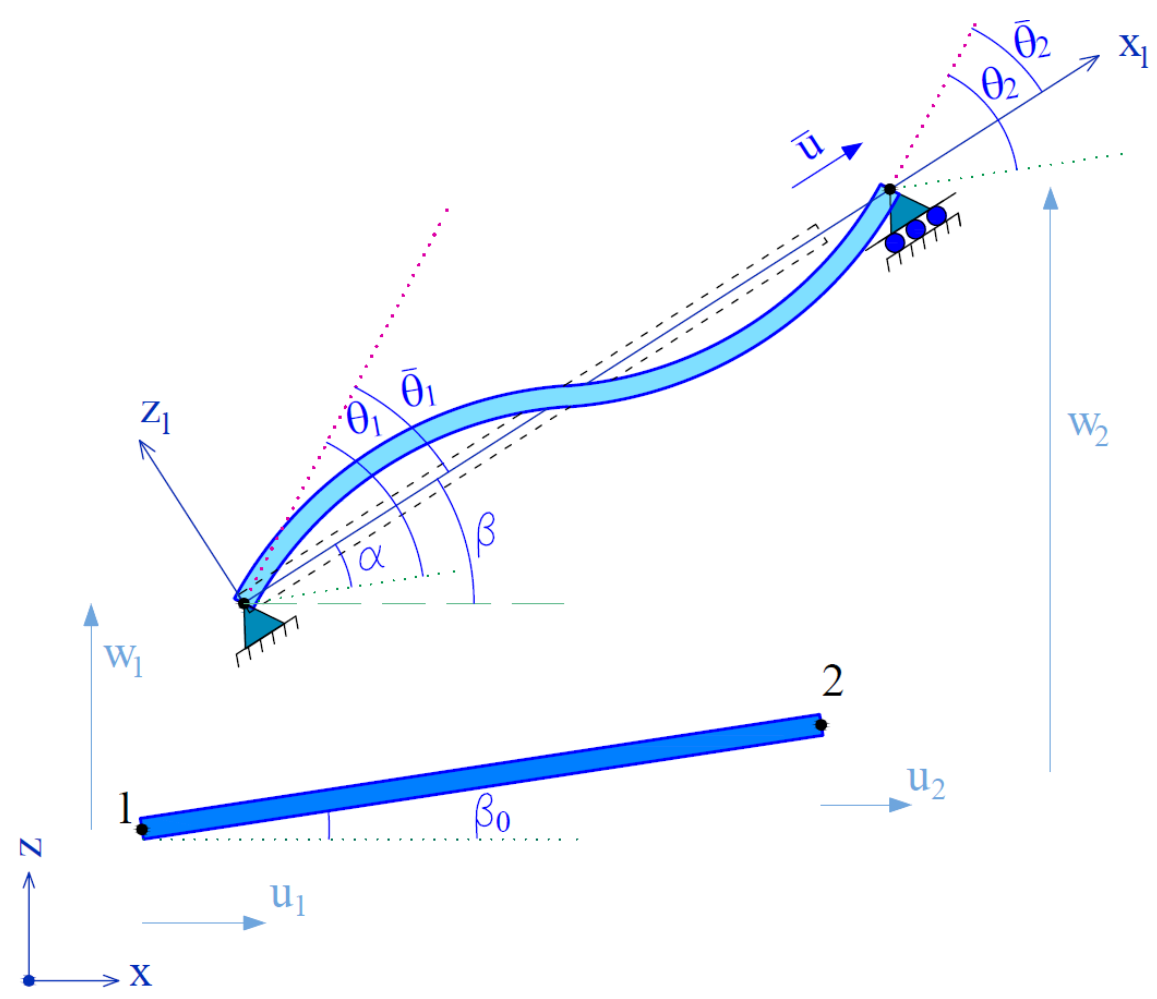

Figure 2. Corotacional Fomulation - beam kinematics.

Furthermore, the local displacement vector is given by:

$$
\boldsymbol{u}_{\boldsymbol{l}}=\left\{\begin{array}{lll}
\bar{u} & \bar{\theta}_{1} & \bar{\theta}_{2}
\end{array}\right\}^{T} .
$$

The $\boldsymbol{u}_{\boldsymbol{l}}$ components can be calculated by the following equations,

$$
\bar{u}=l_{n}-l_{0}, \quad \bar{\theta}_{1}=\theta_{1}-\alpha \quad \text { and } \quad \bar{\theta}_{2}=\theta_{2}-\alpha .
$$

In the equations above $l_{0}$ and $l_{n}$ denote the initial and current lengths, respectively. Using simple trigonometric relationships the lengths may be rewritten as:

$$
l_{0}=\left[\left(x_{2}-x_{1}\right)^{2}-\left(z_{2}-z_{1}\right)^{2}\right]^{1 / 2} \text { and } l_{n}=\left[\left(x_{2}+u_{2}-x_{1}-u_{1}\right)^{2}-\left(z_{2}+w_{2}-z_{1}-w_{1}\right)^{2}\right]^{1 / 2}
$$

where $\alpha$ denotes the rigid body rotation. This may be related trigonometrically by

$$
\operatorname{sen} \alpha=c_{0} s-s_{0} c \quad \text { and } \cos \alpha=c_{0} c+s_{0} s,
$$

which, after developed, leads:

$$
\begin{gathered}
c_{0}=\cos \beta_{0}=\frac{1}{l_{0}}\left(x_{2}-x_{1}\right), \quad s_{0}=\operatorname{sen} \beta_{0}=\frac{1}{l_{0}}\left(z_{2}-z_{1}\right), \\
c=\cos \beta=\frac{1}{l_{n}}\left(x_{2}+u_{2}-x_{1}-u_{1}\right) \text { and } \quad s=\operatorname{sen} \beta=\frac{1}{l_{n}}\left(z_{2}+w_{2}-z_{1}-w_{1}\right) .
\end{gathered}
$$


Thus, for $|\alpha|<\pi, \alpha$ is given by:

$\alpha=\operatorname{sen}^{-1}(\operatorname{sen} \alpha)$ if $\operatorname{sen} \alpha \geq 0$ and $\cos \alpha \geq 0 ; \alpha=\cos ^{-1}(\cos \alpha)$ if $\operatorname{sen} \alpha \geq 0$ and $\cos \alpha<0$

$\alpha=\operatorname{sen}^{-1}(\operatorname{sen} \alpha)$ if $\operatorname{sen} \alpha<0$ and $\cos \alpha \geq 0$ and $\alpha=-\cos ^{-1}(\cos \alpha)$ if $\operatorname{sen} \alpha<0$ and $\cos \alpha<0$ (7)

\subsubsection{Virtual displacements}

Deriving the equation (3) obtains the virtual local displacements,

$$
\begin{aligned}
& \delta \bar{u}=c\left(\delta u_{2}-\delta u_{1}\right)+s\left(\delta w_{2}-\delta w_{1}\right)=\left\{\begin{array}{llllll}
-c & -s & 0 & c & s & 0
\end{array}\right\} \delta \boldsymbol{u}_{g} \\
& \delta \bar{\theta}_{1}=\delta \theta_{1}-\delta \alpha=\delta \theta_{1}-\delta \beta \quad\left(\alpha=\beta-\beta_{0}\right) \quad \text { and } \\
& \delta \bar{\theta}_{2}=\delta \theta_{2}-\delta \alpha=\delta \theta_{2}-\delta \beta
\end{aligned}
$$

On the other hand, $\delta \beta$ can be calculated by differentiation of Equation (6d)

$$
\delta \beta=\frac{1}{c l_{n}^{2}}\left[\left(\delta w_{2}-\delta w_{1}\right) l_{n}-\left(z_{2}+w_{2}-z_{1}-w_{1}\right) \delta l_{n}\right]
$$

where $\delta l_{n}=\delta \bar{u}$ is given by Equation (8). Using Eq (6d), the expression $\delta \beta$ becomes

$$
\delta \beta=\frac{1}{c l_{n}}\left[\left(\delta w_{2}-\delta w_{1}\right) l_{n}-s c\left(\delta u_{2}-\delta u_{1}\right)-s^{2}\left(\delta w_{2}-\delta w_{1}\right)\right]
$$

which, after simplifications produces

$$
\delta \beta=\frac{1}{l_{n}}\left\{\begin{array}{llllll}
-c & -S & 0 & c & s & 0
\end{array} \delta \boldsymbol{u}_{g} .\right.
$$

Thus, the transformation matrix $\boldsymbol{B}$ is defined as

$$
\delta \boldsymbol{u}_{\boldsymbol{l}}=\boldsymbol{B} \delta \boldsymbol{u}_{\mathrm{g}}
$$

and given by,

$$
\boldsymbol{B}=\left[\begin{array}{cccccc}
-c & -s & 0 & c & s & 0 \\
-s / l_{n} & c / l_{n} & 1 & s / l_{n} & -c / l_{n} & 0 \\
-s / l_{n} & c / l_{n} & 0 & s / l_{n} & -c / l_{n} & 1
\end{array}\right]
$$

\subsubsection{Internal force vector}

The relationship between the internal local force vector $f_{l}$ and global $f_{g}$ is obtained by the equation of virtual work in local and global systems 


$$
V=\delta \boldsymbol{u}_{g}^{T} \boldsymbol{f}_{g}=\delta \boldsymbol{u}_{l}^{T} \boldsymbol{f}_{l}=\delta \boldsymbol{u}_{g}^{T} \boldsymbol{B}^{T} \boldsymbol{f}_{l}
$$

Equation (16) must be applied to any $\delta \boldsymbol{u}_{g}$ arbitrary. Thus, the global internal force vector is given by

$$
\boldsymbol{f}_{g}=\boldsymbol{B}^{T} \boldsymbol{f}_{l}
$$

in which the local internal force vector $f_{l}=\left\{\begin{array}{lll}N & M_{1} & M_{2}\end{array}\right\}^{T}$ depends on the definition of the finite element beam specific employed.

\subsubsection{Tangent stiffness matrix}

The global tangent stiffness matrix $\boldsymbol{K}_{g}$ defined by

$$
\delta \boldsymbol{f}_{g}=\boldsymbol{K}_{g} \delta \boldsymbol{u}_{g}
$$

is obtained by variation of Equation (17). Thus,

$$
\delta \boldsymbol{f}_{g}=\boldsymbol{B}^{T} \delta \boldsymbol{f}_{l}+N \delta b_{1}+M_{1} \delta b_{2}+M_{2} \delta b_{3} .
$$

In the above equation $\boldsymbol{b}_{2}$ is, for example, the second column of $\boldsymbol{B}^{T}$. The following notations are introduced,

$$
\boldsymbol{r}=\left\{\begin{array}{llllll}
-c & -s & 0 & c & s & 0
\end{array}\right\}^{T} \text { and } \quad \boldsymbol{z}=\left\{\begin{array}{llllll}
s & -c & 0 & -s & c & 0
\end{array}\right\}^{T}
$$

which by differentiation become,

$$
\delta \boldsymbol{r}=\boldsymbol{z} \delta \beta \quad \text { and } \quad \delta \boldsymbol{z}=-\boldsymbol{r} \delta \beta
$$

The equation (8) and (13) may be rewritten as

$$
\delta \bar{u}=\delta l_{n}=\boldsymbol{r}^{T} \delta \boldsymbol{u}_{g} \quad \text { and } \quad \delta \beta=\frac{1}{l_{n}} \boldsymbol{z}^{T} \delta \boldsymbol{u}_{g}
$$

Introduces auxiliary expressions,

$$
\boldsymbol{b}_{1}=\boldsymbol{r}, \quad \boldsymbol{b}_{2}=\left\{\begin{array}{llllll}
0 & 0 & 1 & 0 & 0 & 0
\end{array}\right\}^{T}-\frac{1}{l_{n}} \boldsymbol{z} \quad \text { and } \quad \boldsymbol{b}_{3}=\left\{\begin{array}{llllll}
0 & 0 & 0 & 0 & 0 & 1
\end{array}\right\}^{T}-\frac{1}{l_{n}} \boldsymbol{z}
$$

derivatives produce,

$$
\delta \boldsymbol{b}_{1}=\delta \boldsymbol{r}=\frac{\boldsymbol{z z ^ { T }}}{l_{n}} \delta \boldsymbol{p}_{g} \quad \text { and } \quad \delta \boldsymbol{b}_{2}=\delta \boldsymbol{b}_{3}=-\frac{\delta \boldsymbol{z}}{l_{n}}+\frac{\boldsymbol{z} \delta l_{n}}{l_{n}^{2}}=\frac{1}{l_{n}^{2}}\left(\boldsymbol{r} \boldsymbol{z}^{T}+\boldsymbol{z} \boldsymbol{r}^{T}\right) \delta \boldsymbol{p}_{g} .
$$


The first term in Eq (19) is calculated by the introduction of the local tangent stiffness matrix $\boldsymbol{K}_{l}$, which depends on the element definition.

$$
\delta \boldsymbol{f}_{l}=\boldsymbol{K}_{l} \delta \boldsymbol{u}_{l}=\boldsymbol{K}_{l} \boldsymbol{B} \delta \boldsymbol{u}_{g}
$$

Finally, from Equation (18), (19), (25a), (25b) and (26), the expression of the global stiffness matrix tangent becomes

$$
\boldsymbol{K}_{g}=\boldsymbol{B}^{T} \boldsymbol{K}_{l} \boldsymbol{B}+\frac{N}{l_{n}} z z^{T}+\frac{1}{l_{n}^{2}}\left(\boldsymbol{r} z^{T}+z \boldsymbol{r}^{T}\right)\left(M_{1}+M_{2}\right)
$$

The equation (14) and (27) provide the connection between the internal forces and stiffness matrices tangent local and global. These relationships are independent of the local definition of the element. This is obtained by adopting assumptions: Bernoulli deformation, linear elastic constitutive relation, the principle of virtual work (PTV) $V=\int_{v} \sigma \delta \varepsilon d v=N \delta \bar{u}+M_{1} \delta \bar{\theta}_{1}+M_{2} \delta \bar{\theta}_{2}$.

\subsection{Timoshenko beam element}

A classical two node Timoshenko beam element is defined with linear interpolations for $\mathrm{u}, \mathrm{w}$ and $\theta$ in the local co-rotational coordinate system. These are given by:

$$
\begin{aligned}
& u=\frac{x}{L} \bar{u}, \\
& w=0 \text { and } \\
& \theta=\left(1-\frac{x}{L}\right) \bar{\theta}_{1}+\frac{x}{L} \bar{\theta}_{2} .
\end{aligned}
$$

The curvature $k$, shear deformation $\gamma$ and strain $\varepsilon$ are defined by

$$
\begin{aligned}
& k=\frac{\partial \theta}{\partial x}=\frac{\bar{\theta}_{2}-\bar{\theta}_{1}}{L}, \\
& \gamma=\frac{\partial w}{\partial x}-\theta=-\left(1-\frac{x}{L}\right) \bar{\theta}_{1}-\frac{x}{L} \bar{\theta}_{2} \quad \text { and } \\
& \varepsilon=\frac{\partial u}{\partial x}-k z=\frac{\bar{u}}{L}-\frac{\bar{\theta}_{2}-\bar{\theta}_{1}}{L} z .
\end{aligned}
$$

\subsubsection{Local internal force vector}

The local internal forces are calculated using the following assumptions: definition of linear deformation of Timoshenko; linear elastic constitutive relation and; the principle of virtual work, which in this case accounts for shear deformation and is expressed as:

$$
V=\int_{v}(\sigma \delta \varepsilon+\tau \delta \gamma) d v=N \delta \bar{u}+M_{1} \delta \bar{\theta}_{1}+M_{2} \delta \bar{\theta}_{2}
$$


The calculation of $\delta \varepsilon$ and $\delta \gamma$ by differentiation of (29b-c) and its introduction in the previous equation yields,

$$
V=\int_{v}\left\{\frac{\sigma}{L}\left[\delta \bar{u}-z\left(\delta \bar{\theta}_{2}-\delta \bar{\theta}_{1}\right)\right]-\tau\left[\left(1-\frac{x}{L}\right) \delta \bar{\theta}_{1}+\frac{x}{L} \delta \bar{\theta}_{2}\right]\right\} d v
$$

The internal forces are calculated of (30) and (31) with $x=L / 2$ for avoid shear locking, obtaining in this way:

$$
\begin{aligned}
& N=\int_{v} \frac{\sigma}{L} d v=\int_{A} \sigma d A, \\
& M_{1}=\int_{v}\left(\frac{\sigma}{L} z-\frac{\tau}{2}\right) d v=\int_{A} \sigma z d A-\frac{L}{2} \int_{A} \tau d A \text { and } \\
& M_{2}=\int_{v}\left(-\frac{\sigma}{L} z-\frac{\tau}{2}\right) d v=-\int_{A} \sigma z d A-\frac{L}{2} \int_{A} \tau d A .
\end{aligned}
$$

It is a new integration along the depth of the cross section of the element determining the coefficients of internal forces in the same format that was implemented - Figure 3.

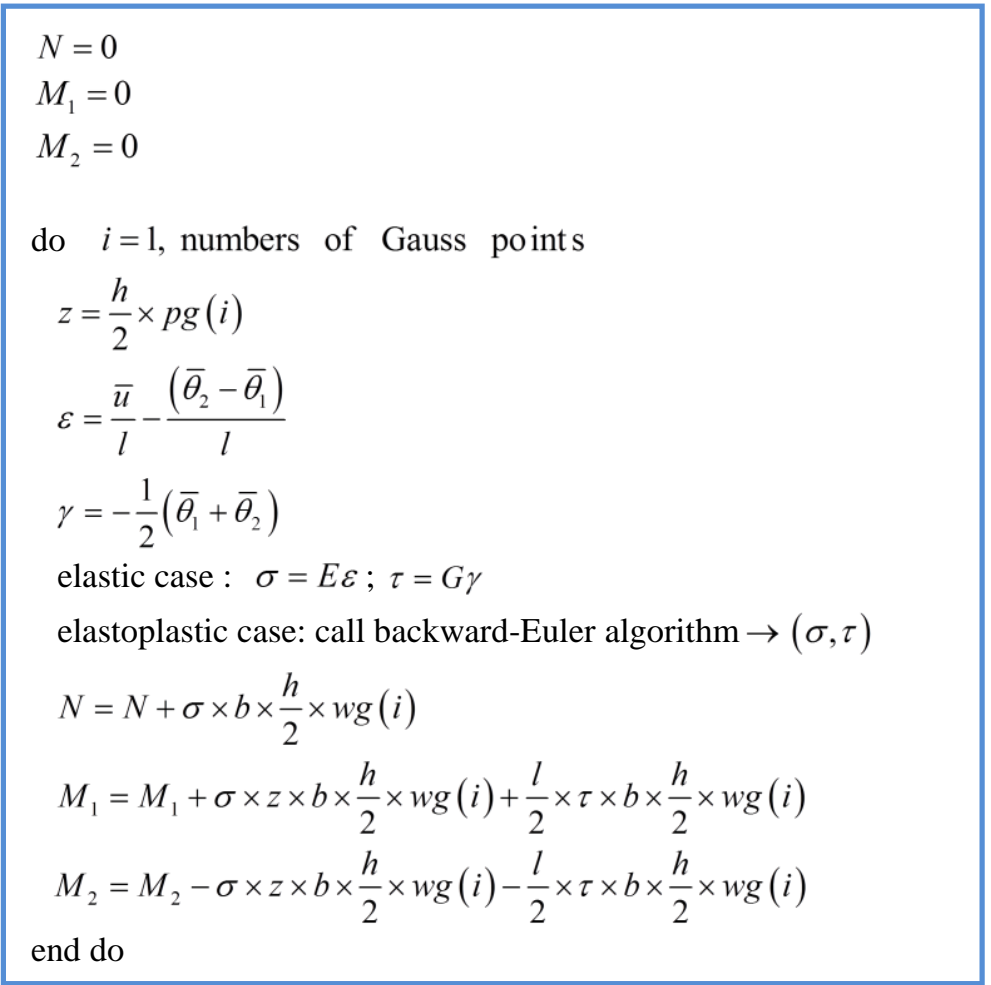

Figure 3. Algorithm to obtain the internal force vector location.

\subsubsection{Local tangent stiffness matrix}

The same assumptions used in the acquisition of the internal force vector are taken. The consistent tangent operator defined by 


$$
\left[\begin{array}{c}
\dot{\sigma} \\
\dot{\tau}
\end{array}\right]=\left[\begin{array}{ll}
C_{t 1} & C_{t 3} \\
C_{t 3} & C_{t 2}
\end{array}\right]\left[\begin{array}{l}
\dot{\varepsilon} \\
\dot{\gamma}
\end{array}\right]
$$

will be seen in Section 3.3. Equation (33) can be rewritten as $\delta \sigma$

$$
\begin{aligned}
& \delta \sigma=C_{t 1} \delta \varepsilon+C_{t 3} \delta \gamma \\
& \delta \tau=C_{t 1} \delta \varepsilon+C_{t 2} \delta \gamma
\end{aligned}
$$

which, by using $(29 b-c)$ gives

$$
\begin{aligned}
& \delta \sigma=\frac{C_{t 1}}{L}\left(\delta \bar{u}+z \delta \bar{\theta}_{1}-z \delta \bar{\theta}_{2}\right)-\frac{C_{t 3}}{2}\left(\delta \bar{\theta}_{1}+\delta \bar{\theta}_{2}\right) \\
& \delta \tau=\frac{C_{t 3}}{L}\left(\delta \bar{u}+z \delta \bar{\theta}_{1}-z \delta \bar{\theta}_{2}\right)-\frac{C_{t 2}}{2}\left(\delta \bar{\theta}_{1}+\delta \bar{\theta}_{2}\right)
\end{aligned}
$$

Differentiation of (32) gives

$$
\begin{aligned}
\delta N & =\int_{A} \delta \sigma d A \\
\delta M_{1} & =\int_{A} \delta \sigma z d A-\frac{L}{2} \int_{A} \delta \tau d A \\
\delta M_{2} & =-\int_{A} \delta \sigma z d A-\frac{L}{2} \int_{A} \delta \tau d A
\end{aligned}
$$

Finally, from (35) and (36), the local tangent stiffness matrix is

$$
\begin{aligned}
K_{11} & =\frac{\partial N}{\partial \bar{u}}=\frac{1}{L} \int_{A} C_{t 1} d A \\
K_{22} & =\frac{\partial M_{1}}{\partial \bar{\theta}_{1}}=\frac{1}{L} \int_{A} C_{t 1} z^{2} d A-\int_{A} C_{t 3} z d A+\frac{L}{4} \int_{A} C_{t 2} d A \\
K_{33} & =\frac{\partial M_{2}}{\partial \bar{\theta}_{2}}=\frac{1}{L} \int_{A} C_{t 1} z^{2} d A-\int_{A} C_{t 3} z d A+\frac{L}{4} \int_{A} C_{t 2} d A \\
K_{12} & =\frac{\partial N}{\partial \bar{\theta}_{1}}=\frac{1}{L} \int_{A} C_{t 1} z d A-\frac{1}{2} \int_{A} C_{t 3} d A \\
K_{13} & =\frac{\partial N}{\partial \bar{\theta}_{2}}=-\frac{1}{L} \int_{A} C_{t 1} z d A-\frac{1}{2} \int_{A} C_{t 3} d A \\
K_{21} & =K_{12} \quad, \quad K_{31}=K_{13} \text { and } K_{32}=K_{23} .
\end{aligned}
$$

Integrating numerically the previous coefficients along the depth of the cross section of the element can be get them in the same format that was implemented (Figure 4). 


$$
\begin{aligned}
k_{11} & =0 \\
k_{12} & =0 \\
k_{13} & =0 \\
k_{22} & =0 \\
k_{23} & =0 \\
k_{33} & =0 \\
\text { do } \quad i= & 1, \text { numbers of Gauss points } \\
y= & \frac{h}{2} \times p g(i) \\
\text { elastic case : } & C_{t 1}=C(1,1)=E \\
C_{t 2} & =C(2,2)=G \\
\text { elastoplastic case: } & C_{t 1}=C(1,1) \\
C_{t 2} & =C(2,2) \\
C_{t 3} & =C(1,2)=C(2,1) \\
k_{11} & =k_{11}+\frac{1}{l} C_{t 1} \times b \times \frac{h}{2} \times w g(i) \\
k_{12} & =k_{12}-\frac{1}{2} C_{t 3} \times b \times \frac{h}{2} \times w g(i) \\
k_{13} & =k_{13}-\frac{1}{2} C_{t 3} \times b \times \frac{h}{2} \times w g(i) \\
k_{22} & =k_{22}+\frac{1}{l} \times C_{t 1} \times z^{2} \times b \times \frac{h}{2} \times w g(i)+\frac{l}{4} \times C_{t 2} \times b \times \frac{h}{2} \times w g(i) \\
k_{23} & =k_{23}-\frac{1}{l} \times C_{t 1} \times z^{2} \times b \times \frac{h}{2} \times w g(i)+\frac{l}{4} \times C_{t 2} \times b \times \frac{h}{2} \times w g(i) \\
k_{33} & =k_{33}+\frac{1}{l} \times C_{t 1} \times z^{2} \times b \times \frac{h}{2} \times w g(i)+\frac{l}{4} \times C_{t 2} \times b \times \frac{h}{2} \times w g(i) \\
\text { end do } &
\end{aligned}
$$

Figure 4: Algorithm to obtain the tangent stiffness matrix.

\subsection{Constitutive equations}

In the plastic rate equations for the Timoshenko beam two strains $(\varepsilon, \gamma)$ and two stresses $(\sigma, \tau)$ are involved. There are many different algorithms to integrate these equations and among the iterative procedures, the most popular one is the backward-Euler scheme. It takes a simple form in case of the von Mises yield criterion and it allows the generation of a consistent tangent operator which maintains the quadratic convergence of the Newton-Raphson method. The formulation descript here is mainly taken from Battini [2].

\subsubsection{Plane beam equations}

The relations for the plane beam are derived from the von Mises material with isotropic hardening under plane stress conditions [3] by setting 


$$
\sigma_{z}=0
$$

The von Mises yield function is

$$
f=\left(\sigma_{x}^{2}+3 \tau_{x z}^{2}\right)^{1 / 2}-\sigma_{0}\left(\varepsilon_{p s}\right)=\sigma_{e}-\sigma_{0}\left(\varepsilon_{p s}\right)
$$

with

$$
\varepsilon_{p s}=\int \dot{\varepsilon}_{p s} d t \quad \dot{\varepsilon}_{p s}=\frac{2}{\sqrt{3}}\left(\dot{\varepsilon}_{p x}^{2}+\dot{\varepsilon}_{p z}^{2}+\dot{\varepsilon}_{p x} \dot{\varepsilon}_{p x}+\dot{\gamma}_{p x z}^{2} / 4\right)=\dot{\lambda}
$$

The hardening parameter $H$ is calculated from the uniaxial stress-strain law

$$
H=\frac{\partial \sigma_{0}}{\partial \varepsilon_{p s}}=\frac{E_{t}}{1-E_{t} / E}
$$

which gives

$$
\sigma_{0}=\sigma_{Y}+H \varepsilon_{p s}
$$

where $\sigma_{Y}$ is the yield stress. The Prandtl-Reuss flow rules associated to (39) are

$$
\dot{\varepsilon}_{p}=\left[\begin{array}{c}
\dot{\varepsilon}_{p x} \\
\dot{\varepsilon}_{p z} \\
\dot{\gamma}_{p x z}
\end{array}\right]=\dot{\lambda} \frac{\partial f}{\partial \boldsymbol{\sigma}}=\dot{\lambda} \boldsymbol{a}=\frac{\dot{\lambda}}{2 \sigma_{e}}\left[\begin{array}{c}
2 \sigma_{x} \\
-\sigma_{x} \\
6 \tau_{x z}
\end{array}\right]
$$

The stress changes are related to the strain changes via

$$
\dot{\boldsymbol{\sigma}}_{0}=\boldsymbol{C}\left(\dot{\boldsymbol{\varepsilon}}-\dot{\boldsymbol{\varepsilon}}_{p}\right)=\boldsymbol{C}(\dot{\boldsymbol{\varepsilon}}-\dot{\lambda} \boldsymbol{a})
$$

where

$$
\boldsymbol{C}=\frac{E}{1-v^{2}}\left[\begin{array}{ccc}
1 & v & 0 \\
v & 1 & 0 \\
0 & 0 & (1-v) / 2
\end{array}\right] \quad \dot{\boldsymbol{\sigma}}=\left[\begin{array}{c}
\dot{\sigma}_{x} \\
\dot{\sigma}_{z} \\
\dot{\tau}_{x z}
\end{array}\right] \quad \dot{\boldsymbol{\varepsilon}}=\left[\begin{array}{c}
\dot{\varepsilon}_{x} \\
\dot{\varepsilon}_{z} \\
\dot{\gamma}_{x z}
\end{array}\right]
$$

By assuming that the stresses must remain on the yield surface in case of plastic loading $(\dot{\lambda}>0)$, it is obtained

$$
\dot{f}=\frac{\partial f^{T}}{\partial \boldsymbol{\sigma}} \dot{\boldsymbol{\sigma}}+\frac{\partial f}{\partial \sigma_{0}} \frac{\partial \sigma_{0}}{\partial \varepsilon_{p s}} \dot{\boldsymbol{\varepsilon}}_{p s}=\boldsymbol{a}^{T} \dot{\boldsymbol{\sigma}}-H \dot{\lambda}=0
$$

which by using (44) gives

$$
\dot{\lambda}=\frac{\boldsymbol{a}^{T} \boldsymbol{C} \dot{\boldsymbol{\varepsilon}}}{\boldsymbol{a}^{T} \boldsymbol{C a}+H}
$$


Since $\dot{\sigma}_{Z}=0$, the second equation of (44) gives

$$
\dot{\varepsilon}_{z}=-v \dot{\varepsilon}_{x}+\frac{\dot{\lambda} \sigma_{x}}{\sigma_{e}}\left(v-\frac{1}{2}\right)
$$

which by introduction in the first and third equations of (44) gives

$$
\dot{\sigma}_{x}=E\left(\dot{\varepsilon}_{x}-\frac{\sigma_{x}}{\sigma_{e}} \dot{\lambda}\right) \quad \dot{\tau}_{x z}=G\left(\dot{\gamma}_{x z}-\frac{3 \tau_{x z}}{\sigma_{e}} \dot{\lambda}\right)
$$

Substitution of equations (48) and (49) in (47) gives after some algebra

$$
\dot{\lambda}=\frac{\sigma_{e}\left(E \sigma_{x} \dot{\varepsilon}_{x}+3 G \tau_{x z} \dot{\gamma}_{x z}\right)}{\left(E \sigma_{x}^{2}+9 G \tau_{x z}^{2}\right)+H \sigma_{e}^{2}}
$$

Finally, introducing (47) in (44) gives

$$
\dot{\boldsymbol{\sigma}}=\boldsymbol{C}_{t} \dot{\boldsymbol{\varepsilon}}=\boldsymbol{C}\left(\boldsymbol{I}-\frac{\boldsymbol{a a ^ { T }} \boldsymbol{C}}{\boldsymbol{a C a ^ { T } + H}}\right) \dot{\boldsymbol{\varepsilon}}
$$

where $C_{t}$ is the tangent operator. The backward-Euler algorithm shown in Figure 6 consists in applying an elastic forward step ( $A B$ ) followed by a return mapping ( $B C$ ) on the yield surface - Figure 5 .

$$
\sigma_{C}=\sigma_{B}-\Delta \lambda C a_{C}=\sigma_{A}+C \Delta \varepsilon-\Delta \lambda C a_{C}
$$

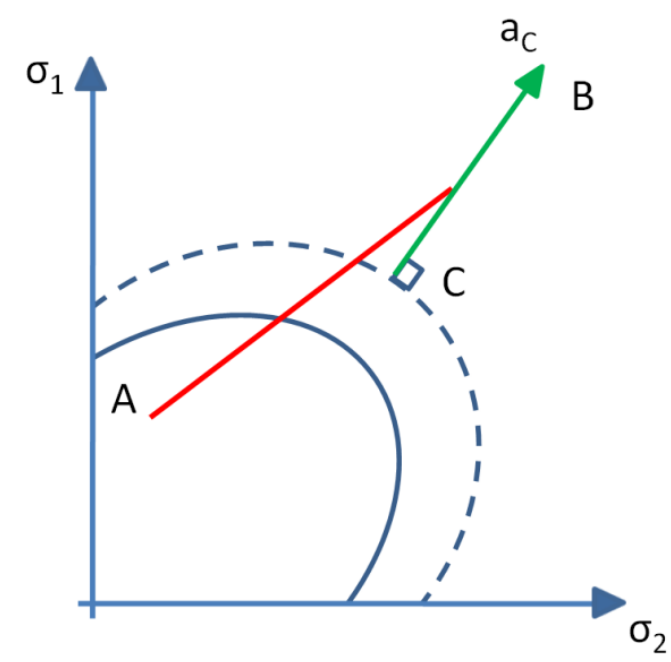

Figure 5: Bacward-Euler scheme.

The vector $\boldsymbol{a}_{C}$ is normal to the yield surface at $C$, which is not known and therefore an iterative procedure must be used. Since $\sigma_{z C}=\sigma_{z A}=0$, the second equation of (52) gives 


$$
\Delta \varepsilon_{z}=-v \Delta \varepsilon_{x}+\frac{\Delta \lambda \sigma_{x C}}{2 \sigma_{e C}}(2 v-1)
$$

which, by substitution into the first and third equations, gives after some algebra

$$
\begin{aligned}
& \sigma_{x C}-\sigma_{x A}=E\left(\Delta \varepsilon_{z}-\frac{\sigma_{x C}}{\sigma_{e C}} \Delta \lambda\right) \\
& \tau_{x z C}-\tau_{x z A}=E\left(\Delta \gamma_{x z}-\frac{3 G \tau_{x z C}}{\sigma_{e C}} \Delta \lambda\right)
\end{aligned}
$$

By introducing the notations

$$
\sigma=\sigma_{x} \quad \tau=\tau_{x z} \quad \gamma=\gamma_{x z}
$$

and new definitions for $\boldsymbol{C}, \boldsymbol{\sigma}, \boldsymbol{\varepsilon}, \boldsymbol{a}$

$$
\boldsymbol{C}=\left[\begin{array}{cc}
E & 0 \\
0 & G
\end{array}\right] \quad \boldsymbol{\sigma}=\left[\begin{array}{l}
\sigma \\
\tau
\end{array}\right] \quad \boldsymbol{\varepsilon}=\left[\begin{array}{l}
\varepsilon \\
\gamma
\end{array}\right]
$$

equations (43), (49), (50) and (54) can be combined and rewritten as

$$
\begin{gathered}
\dot{\boldsymbol{\varepsilon}}_{p}=\dot{\lambda} \boldsymbol{a}=\frac{\dot{\lambda}}{\sigma_{e}}\left[\begin{array}{c}
\sigma \\
3 \tau
\end{array}\right] \\
\dot{\boldsymbol{\sigma}}=\boldsymbol{C}\left(\dot{\boldsymbol{\varepsilon}}-\dot{\boldsymbol{\varepsilon}}_{p}\right)=\boldsymbol{C}(\dot{\boldsymbol{\varepsilon}}-\dot{\lambda} \boldsymbol{a}) \\
\dot{\lambda}=\frac{\boldsymbol{a}^{T} \boldsymbol{C} \dot{\boldsymbol{\varepsilon}}}{\boldsymbol{a}^{T} \boldsymbol{C a}+H} \\
\boldsymbol{\sigma}_{C}=\boldsymbol{\sigma}_{B}-\Delta \lambda \boldsymbol{C} \boldsymbol{a}_{C}=\boldsymbol{\sigma}_{A}+\boldsymbol{C} \Delta \varepsilon-\Delta \lambda \boldsymbol{C} \boldsymbol{a}_{C}
\end{gathered}
$$

It is therefore proved by comparing (44), (47) and (52) with (58), (59) and (60) that the equations for the plane beam can be written in the same form as those under plane stress conditions. It can be noted that the elastic forward step in the backward-Euler scheme does not give $\sigma_{z B}=0$, but the Equation (60) proves that $\sigma_{z B}$ does not need to be calculated.

\subsubsection{Backward-Euler scheme}

The algorithm of the backward-Euler scheme is taken from Battini [2]. The first estimation of $\sigma_{C}$ is calculated with

$$
\sigma_{C}=\sigma_{B}-\Delta \lambda C a_{B}=\sigma_{A}+C \Delta \varepsilon-\Delta \lambda C a_{B}
$$

where $\boldsymbol{a}_{B}$ is calculated from (57) and $\Delta \lambda$ is determined from a first order Taylor expansion of the yield function around point $B$ 


$$
f=f_{B}+\frac{\partial f^{T}}{\partial \boldsymbol{\sigma}} \Delta \boldsymbol{\sigma}_{B}+\frac{\partial f}{\partial \sigma_{0}} \frac{\partial \sigma_{0}}{\partial \varepsilon_{p s}} \Delta \varepsilon_{p s}=f_{B}+\boldsymbol{a}_{B}^{T} \Delta \boldsymbol{\sigma}_{B}-H \Delta \lambda
$$

$\Delta \boldsymbol{\sigma}_{B}$ is the calculated from (58) as

$$
\Delta \boldsymbol{\sigma}_{B}=\boldsymbol{C}\left(\Delta \varepsilon-\Delta \lambda \boldsymbol{a}_{B}\right)
$$

with $\Delta \boldsymbol{\varepsilon}=\boldsymbol{0}$ since the total strain has already been applied in the elastic step $(A B)$. Introducing (62) in (63) and setting $f=0$ gives

$$
\Delta \lambda=\frac{f_{B}}{\boldsymbol{a}_{B}^{T} \boldsymbol{C} \boldsymbol{a}_{B}+H}
$$

Equation (61) gives stresses which do not satisfy the yield function since the normal at $B$ is not the same as the normal at the final position $C$. The iterative process is performed by introducing the vector $\boldsymbol{r}$ defined by the difference between the current stresses and the backward-Euler ones

$$
\boldsymbol{r}=\boldsymbol{\sigma}_{C}-\left(\boldsymbol{\sigma}_{B}-\Delta \lambda \boldsymbol{C} \boldsymbol{a}_{C}\right)
$$

A truncated Taylor expansion of (65) gives

$$
\boldsymbol{r}=\boldsymbol{r}_{0}+\dot{\boldsymbol{\sigma}}+\dot{\lambda} \boldsymbol{C a}+\Delta \lambda \boldsymbol{C} \frac{\partial \boldsymbol{a}}{\partial \boldsymbol{\sigma}} \dot{\boldsymbol{\sigma}}
$$

where

$$
\frac{\partial \boldsymbol{a}}{\partial \boldsymbol{\sigma}}=\frac{\partial^{2} f}{\partial \boldsymbol{\sigma}^{2}}=\frac{3}{\sigma_{e}^{3}}\left[\begin{array}{cc}
\tau^{2} & -\sigma \tau \\
-\sigma \tau & \sigma^{2}
\end{array}\right]
$$

and $\dot{\sigma}$ is the change in $\sigma_{C}$ and $\dot{\lambda}$ is the change in $\Delta \lambda$. The subscript $C$ is from now dropped in order to simplify the notations (all the notations without subscripts refer to $C$ ). Setting $\boldsymbol{r}$ to zero in (66) gives

$$
\dot{\boldsymbol{\sigma}}=-\left(\boldsymbol{I}-\Delta \lambda \boldsymbol{C} \frac{\partial \boldsymbol{a}}{\partial \boldsymbol{\sigma}}\right)^{-1}\left(\boldsymbol{r}_{0}+\dot{\lambda} \boldsymbol{C a}\right)=-\boldsymbol{Q}^{-1}\left(\boldsymbol{r}_{0}+\dot{\lambda} \boldsymbol{C a}\right)
$$

A truncated Taylor expansion of the yield function at $C$ gives

$$
f=f_{0}+\frac{\partial f^{T}}{\partial \boldsymbol{\sigma}} \dot{\boldsymbol{\sigma}}+\frac{\partial f}{\partial \sigma_{0}} \frac{\partial \sigma_{0}}{\partial \varepsilon_{p s}} \dot{\boldsymbol{\varepsilon}}_{p s}=f_{0}+\boldsymbol{a}_{B}^{T} \dot{\boldsymbol{\sigma}}-H \dot{\lambda}
$$

further, setting $f=0$ and using (68) gives

$$
\dot{\lambda}=\frac{f_{0}-\boldsymbol{a}^{T} \boldsymbol{Q}^{-1} r_{0}}{\boldsymbol{a}^{T} \boldsymbol{Q}^{-1} \boldsymbol{a}+H}
$$




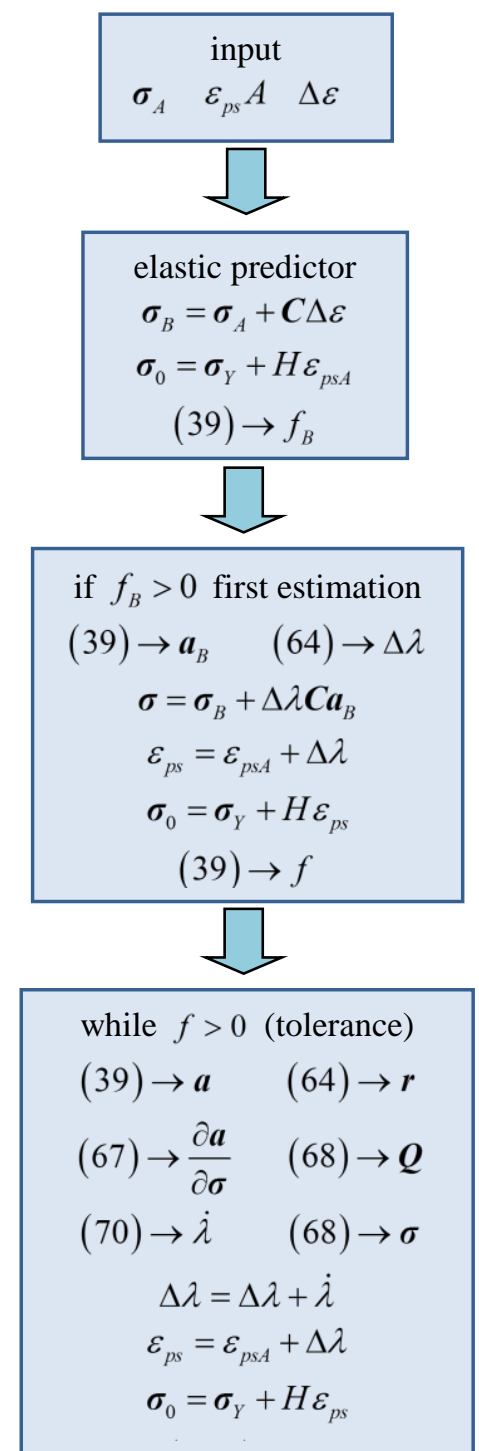

Figure 6. Backward-Euler algorithm.

\subsubsection{Consistent tangent operator}

The tangent operator $\boldsymbol{C}_{t}$ assumes infinitesimal strain and stress changes. However, in order to eliminate numerical elastic unloading, a path-independent strategy is adopted. In order to maintain the quadratic convergence inherent in the Newton-Raphson method, a tangent operator $\boldsymbol{C}_{c t}$, consistent with the backward-Euler scheme is derived [3]. Differentiation of (60) gives (subscript $C$ is dropped)

$$
\dot{\boldsymbol{\sigma}}=\boldsymbol{C} \dot{\boldsymbol{\varepsilon}}-\dot{\lambda} \boldsymbol{C a}-\Delta \lambda \boldsymbol{C} \frac{\partial \boldsymbol{a}}{\partial \boldsymbol{\sigma}} \dot{\boldsymbol{\sigma}}
$$

where the last term in equation (71) is omitted in the derivation of the standard tangent operator. From (71), it is obtained

$$
\dot{\boldsymbol{\sigma}}=\left(\boldsymbol{I}+\Delta \lambda \boldsymbol{C} \frac{\partial \boldsymbol{a}}{\partial \boldsymbol{\sigma}}\right)^{-1} \boldsymbol{C}(\dot{\boldsymbol{\varepsilon}}-\dot{\lambda} \boldsymbol{a})=\boldsymbol{R}(\dot{\boldsymbol{\varepsilon}}-\dot{\lambda} \boldsymbol{a})
$$


The stresses must remain on the yield surface, which, from (46), gives

$$
\dot{f}=a^{T} \dot{\boldsymbol{\sigma}}-H \dot{\lambda}=a^{T}+\boldsymbol{R}(\dot{\boldsymbol{\varepsilon}}-\dot{\lambda} \boldsymbol{a})-H \dot{\lambda}=0
$$

and hence

$$
\dot{\lambda}=\frac{\boldsymbol{a}^{T} \boldsymbol{R} \dot{\boldsymbol{\varepsilon}}}{\boldsymbol{a}^{T} \boldsymbol{R} \boldsymbol{a}+H}
$$

Introducing (74) in (72) finally gives

$$
\dot{\boldsymbol{\sigma}}=\boldsymbol{C}_{c t} \dot{\boldsymbol{\varepsilon}}=\boldsymbol{R}\left(\boldsymbol{I}+\frac{\boldsymbol{a} \boldsymbol{a}^{T} \boldsymbol{R}}{\boldsymbol{a}^{T} \boldsymbol{R} \boldsymbol{a}+H}\right) \dot{\boldsymbol{\varepsilon}}
$$

\section{NUMERICAL EXAMPLES}

\subsection{Lee's frame}

The loading conditions, boundary, geometry and material parameters of structure are shown in Figure 4. This frame was shown by Lee [9] for analysis of large displacement and stability of elastic frames. Battini [2], in turn, used to study beam elements co-rotational instability problems. The equilibrium trajectories of the structure for linear analysis are known in the literature as snap-back in the degree of freedom (DOF) $u$ and snap-through in the DOF $v$, so there are two points limit in the load-displacement diagram - Figure 7.

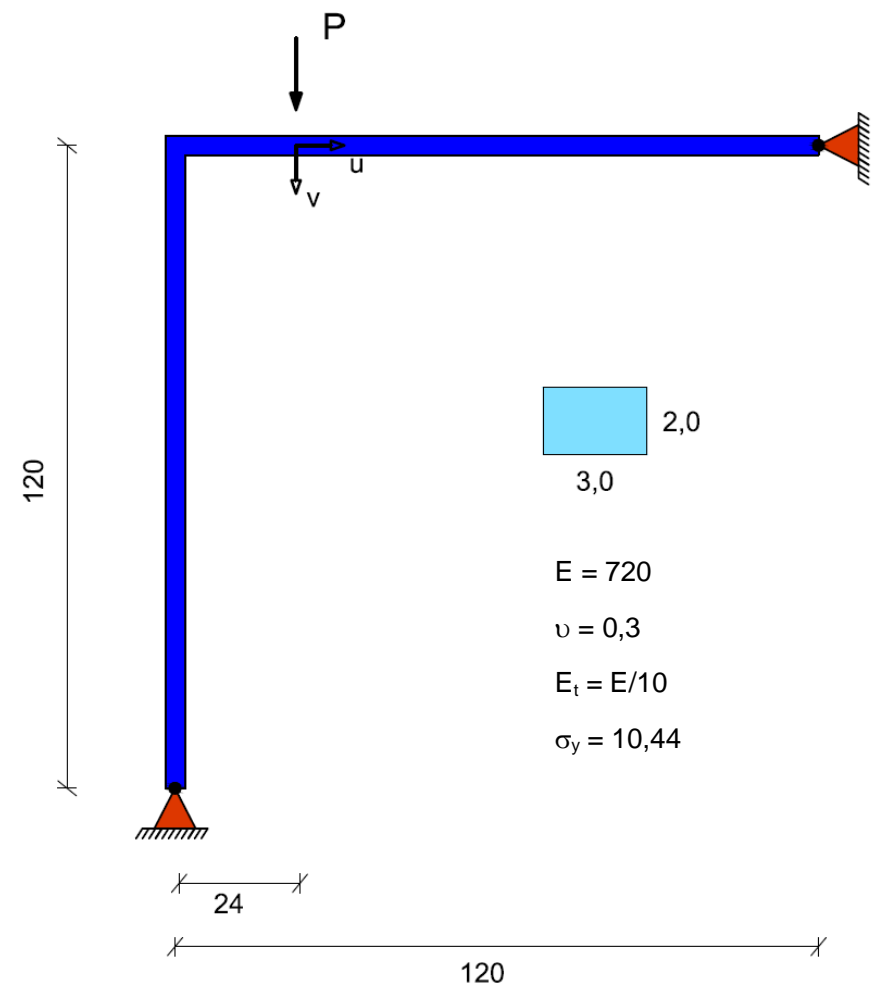

Figure 7. Geometry, boundary conditions and physical parameters. 
It is shown in the Figure 8 that the results are practically the same when using points 7 or 15 Gauss along the depth of the beam. The elastoplastic response (Figure 9 above) in turn both the DOF $\mathrm{u}$ and $\mathrm{v}$ have the snap-through behavior. As we can see, there is an excellent convergence of the results obtained in this study with those obtained by Battini [2].

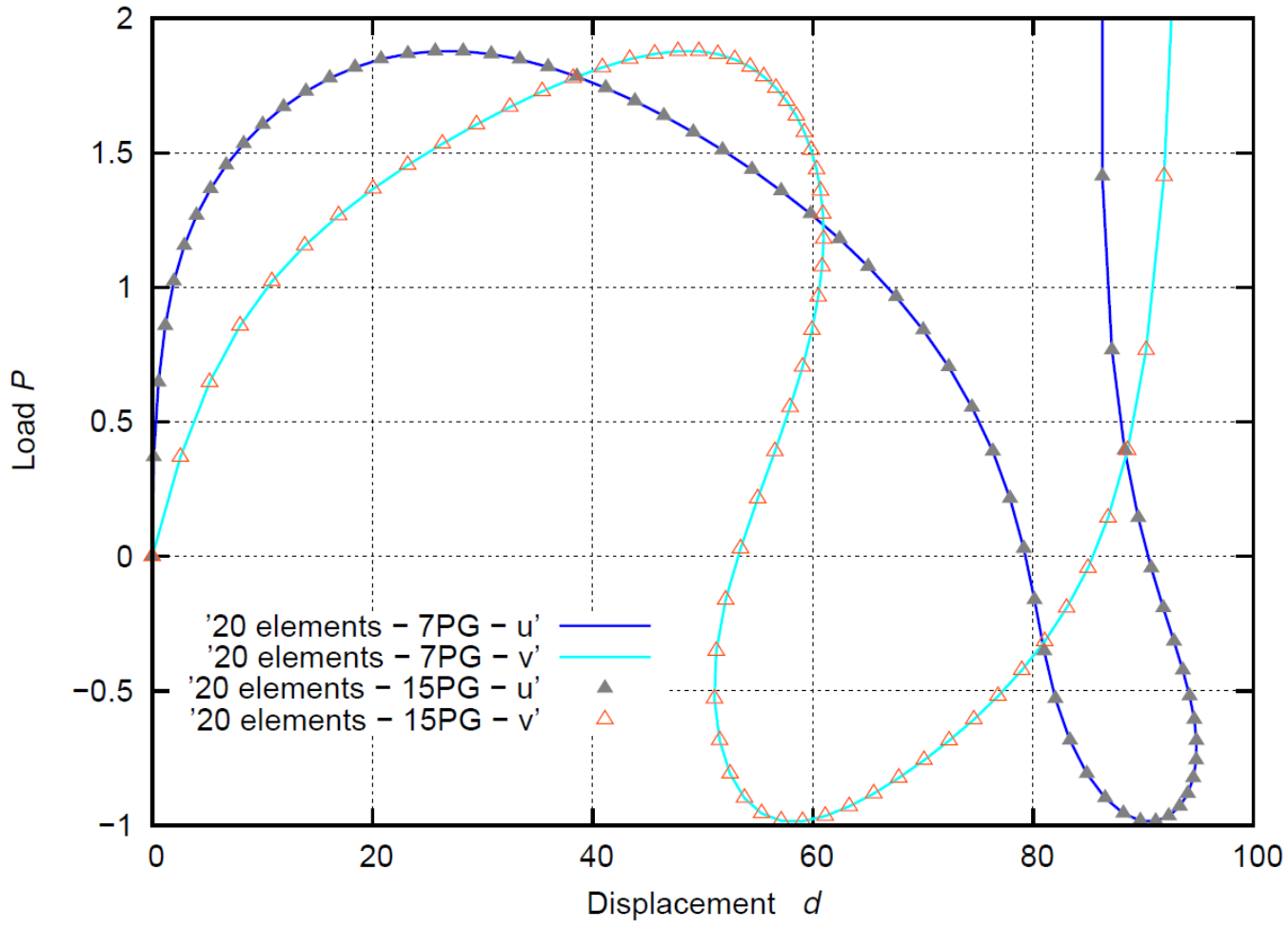

Figure 8. Elastic response - mesh with 20 elements - 7 and 15 Gauss points.

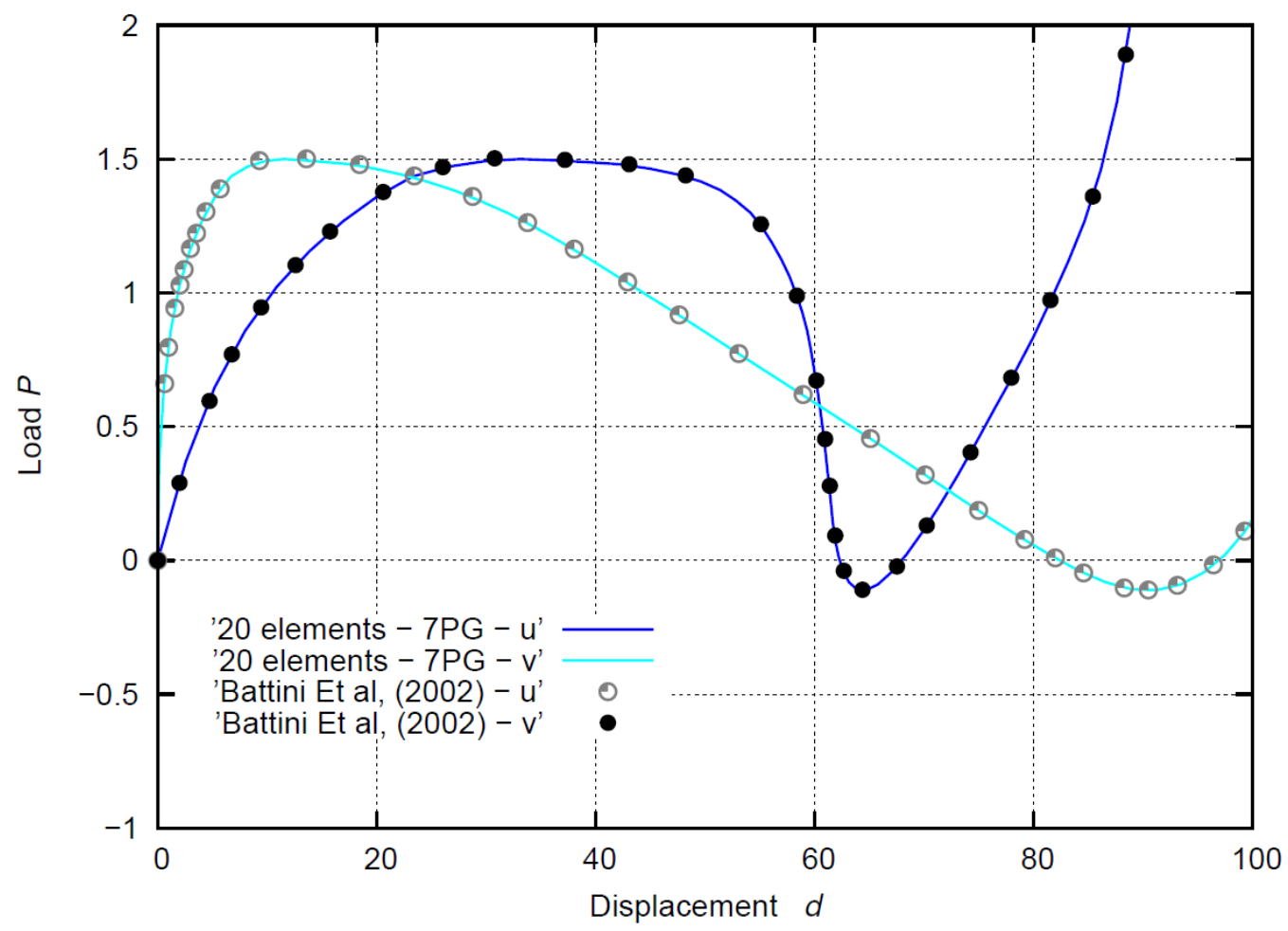

Figure 9. Elastoplastic response - mesh with 20 elements - 7 Gauss points. 


\subsection{Williams toggle frame}

In this work it was used an equivalent rectangular section (with the same inertia and area) to simulate a circular section. The loading conditions, geometric (real and equivalent), boundary, and the material properties of toggle frame studied here can be seen in Figure 10. It was originally solved analytical and experimentally by Williams [12]. Remo [4] also studied in the investigation of large displacements experienced by inelastic frames.

As can be seen by snap-through elastic and elastoplastic paths (Figure 10), although they have the same behavior, the responses of this analysis are less stiffness than those obtained by Remo [4].

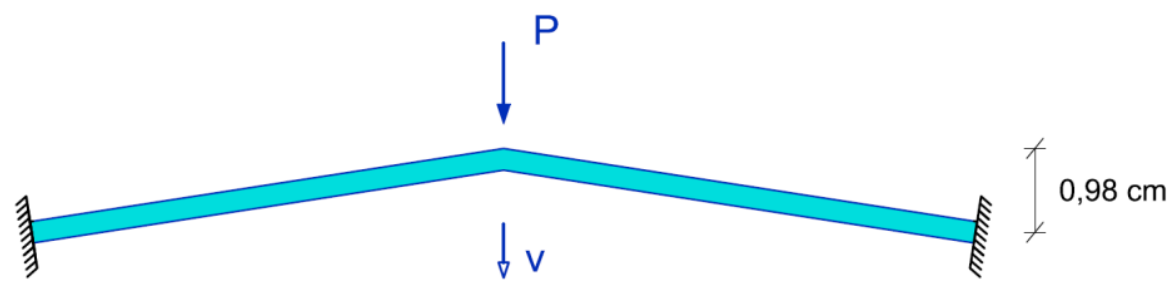

$65,715 \mathrm{~cm}$

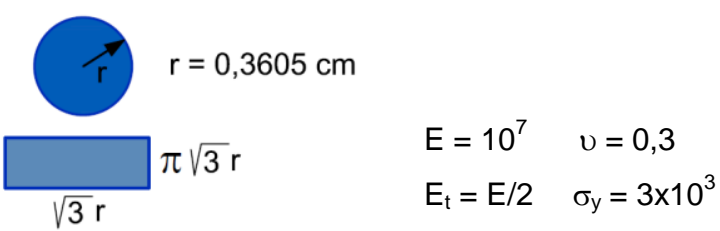

Figure 10: Dimensions, boundary conditions and material properties.

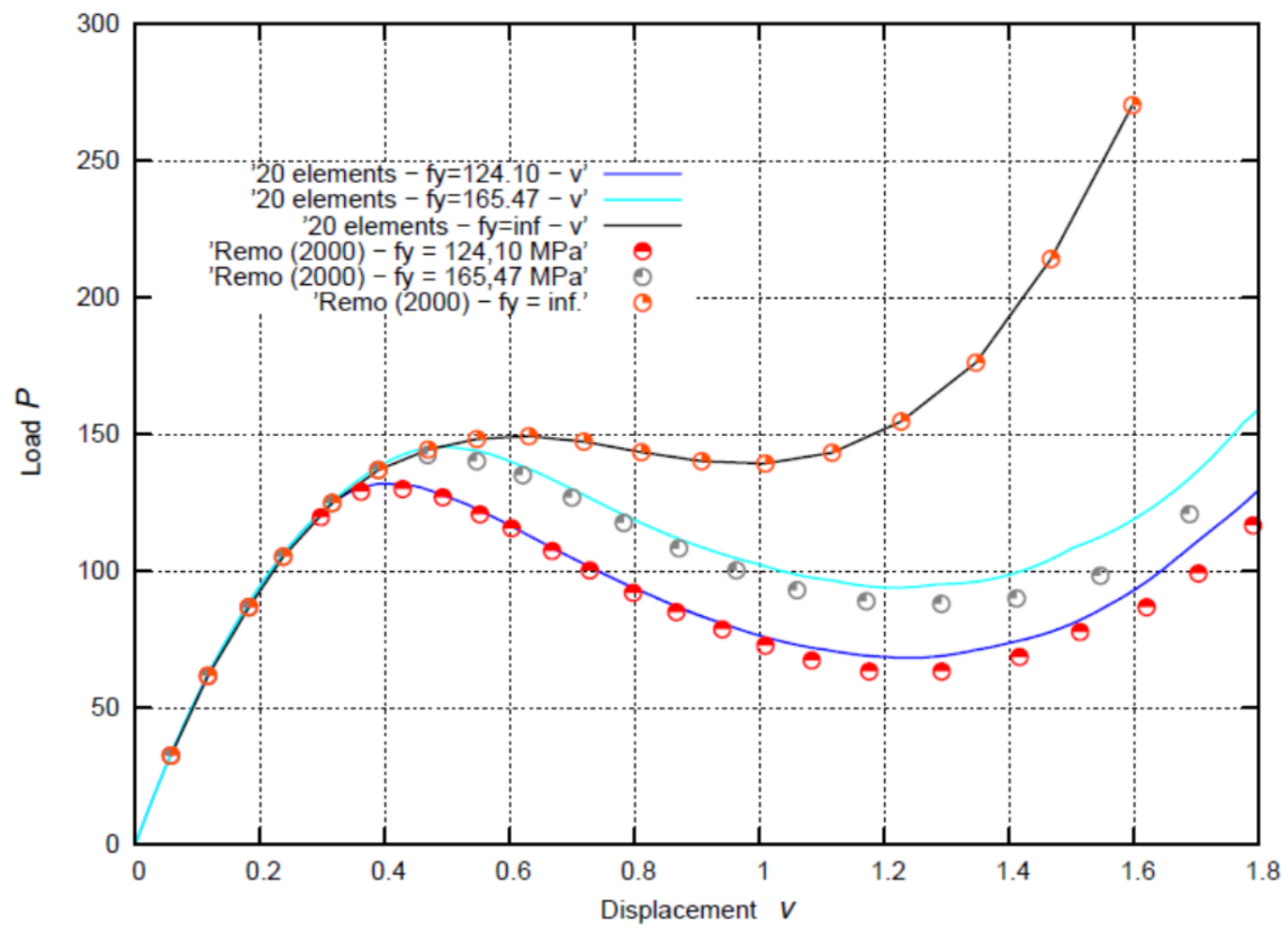

Figure 11: Response elastic and elastoplastic - mesh with 20 elements and 15 Gauss points. 
This fact is probably due to the mixed formulation implemented by this author whereas this article uses a formulation in displacement. Furthermore, in the present work we adopted the hypothesis of an equivalent rectangular section.

\section{CONCLUSIONS}

This study evaluated the efficiency of a beam element 2D Timoshenko applied in a finite element program that takes into account the geometric and physics nonlinearity. In the examples studied - Lee's frames and Williams toggle frames - there is a decrease in bearing capacity of the structure when one considers the phenomenon of elastoplasticity. In addition, highlights the ability of corotational formulation to capture large displacements and rotations.

In elastoplastic analysis, the use of fifteen points Gauss at the time of cross-section instead of seven does not produce a significant improvement in results. Finally, we conclude that the use of simple strategy of equivalent rectangular sections in the study of other types of cross sections provide satisfactory results.

\section{Acknowledgements}

The authors wish to express gratitude for the financial support received from the CNPq for financial support and the Postgraduate Program in Structures and Civil Construction $\mathrm{PECC} / \mathrm{UnB}$.

\section{REFERENCES}

[1] Argyris, J.H., "Continua and discontinua", Proceedings 1st Conference on Matrix Methods in Structural Mechanics, AFFDL-TR-66-80, Air Force Institute of Tecnology, Dayton, Ohio-USA, 1965.

[2] Battini, J.M., "Co-rotational beam elements in instability problems", Ph.D Thesis, Royal Institute of Tecnology - Departament of Mechanics, Stockholm / Sweeden, 2002.

[3] Crisfield, M.A., "Non-linear finite element analysis of solids and structures", Volume 1: Essential, John Wiley \& Sons, Chichester, UK, 1991.

[4] De Souza, R. M., "Force-Based Finite Element for Large Displacement Inelastic Analsysis

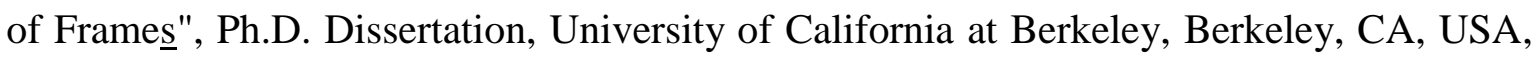
2000.

[5] Felippa, C.A., "Non-linear finite element methods / NFEM", Lecture notes for the course non-linear finite element methods, Center for Aerospace Structures, University of Colorado, Boulder/USA, 2001.

[6] J. N. Reddy., "An Introduction to Nonlinear Finite Element Analysis”, Oxford University Press : Oxford, U.K, 2004.

[7] Owen, D.R.J., Hinton, E., Finite Elements in Plasticity: Theory and Practice, Pineridge. Swansea, U.K, 1980. 
[8] Lemaitre, J., Chaboche, J-L., Mechanics of Solids Materials, Cambrige University Press, Cambrige, , U.K, 1994.

[9] Lee, S. L., Manuel F. S., \& Rossow, E. C., Large deflection analysis and stability of elastic frames. J. Eng. Mech. Div. ASCE 94 EM2, pp. 521-547, 1968.

[10] Menin, R. C. G., "Application of co-rotational description in geometric nonlinear analysis of structures discretized by finite elements of trusses, beams and shells", $\mathrm{PhD}$ thesis, E.TD-004A/06, Brasília : ENC/FT/UnB, 2006.

[11] Simo, J. C., Computational Inelastic, Interdisciplinary Apllied Mathematics, vol. 7, Springer, New York, USA, 1998.

[12] Williams, F. W., 'An approach to the nonlinear behaviour of the members of a rigid jointed plane framework with finite deflection', Quart. J. Mech. Appl. Maths. 17(4), 451469, 1964. 